\title{
Mutagenic and Cytotoxicity LQB 123 Profile: Safety and Tripanocidal Effect of a Phenyl-t-Butylnitrone Derivative
}

\author{
Mauricio Peixoto Cupello, ${ }^{1,2}$ Francis Monique Saraiva, ${ }^{1,2}$ Pedro Ippolito, ${ }^{1}$ \\ Andréia da Silva Fernandes, ${ }^{3}$ Rubem Figueiredo Sadoko Menna-Barreto, ${ }^{4}$ \\ Debora de Sousa dos Santos Costa, ${ }^{5}$ Jessica Isis Oliveira Paula, ${ }^{1,2}$ \\ Paulo Roberto Ribeiro Costa, ${ }^{6}$ Natália Pereira Nogueira, ${ }^{1,2}$ Israel Felzenswalb, ${ }^{3}$ \\ Ayres Guimarães Dias, ${ }^{5}$ and Marcia Cristina Paes ${ }^{1,2,7}$

\footnotetext{
${ }^{1}$ Laboratório de Interação Tripanossomatídeos e Vetores, Departamento de Bioquímica, Instituto de Biologia Roberto Alcantara Gomes, Universidade do Estado do Rio de Janeiro, Rio de Janeiro, RJ, Brazil

${ }^{2}$ Unidade de Desenvolvimento Tecnológico (UDT) de Triagem de Compostos Químicos para Doenças Negligenciadas com Ênfase Farmacológica contra a Doença de Chagas, Universidade do Estado do Rio de Janeiro, Rio de Janeiro, RJ, Brazil

${ }^{3}$ Laboratório de Mutagênese Ambiental LABMUT, Departamento de Biofísica e Biometria, Instituto de Biologia Roberto Alcantara Gomes, Universidade do Estado do Rio de Janeiro, Rio de Janeiro, RJ, Brazil

${ }^{4}$ Laboratório de Biologia Celular, IOC-FIOCRUZ, Rio de Janeiro, RJ, Brazil

${ }^{5}$ Departamento de Química Orgânica, Universidade do Estado do Rio de Janeiro, Rio de Janeiro, RJ, Brazil

${ }^{6}$ Laboratório de Química Bioorgânica, NPPN, Universidade Federal do Rio de Janeiro, Rio de Janeiro, RJ, Brazil

${ }^{7}$ Instituto Nacional de Ciência e Tecnologia, Entomologia Molecular (INCT-EM), Rio de Janeiro, RJ, Brazil
}

Correspondence should be addressed to Marcia Cristina Paes; marcia.paes.uerj@gmail.com

Received 10 September 2016; Accepted 4 January 2017; Published 15 February 2017

Academic Editor: Peter Fu

Copyright (C) 2017 Mauricio Peixoto Cupello et al. This is an open access article distributed under the Creative Commons Attribution License, which permits unrestricted use, distribution, and reproduction in any medium, provided the original work is properly cited.

\begin{abstract}
The therapeutic options for Chagas disease are limited and its treatment presents a number of drawbacks including toxicity, drug resistance, and insufficient effectiveness against the chronic stage of the disease. Therefore, new therapeutical options are mandatory. In the present work, we evaluated the effect of a phenyl-tert-butylnitrone (PBN) derivate, LQB 123, against Trypanosoma cruzi forms. LQB 123 presented a trypanocidal effect against bloodstream trypomastigotes $\left(\mathrm{IC}_{50}=259.4 \pm 6.1 \mu \mathrm{M}\right)$ and intracellular amastigotes infecting peritoneal macrophages $\left(\mathrm{IC}_{50}=188.2 \pm 47.5 \mu \mathrm{M}\right)$, with no harmful effects upon the mammalian cells $\left(\mathrm{CC}_{50}\right.$ values greater than $4 \mathrm{mM})$, resulting in a high selectivity index $\left(\mathrm{CC}_{50} / \mathrm{IC}_{50}>20\right)$. Additionally, metacyclic trypomastigotes submitted to LQB 123 presented an $\mathrm{IC}_{50}$ of about $191.8 \pm 10.5 \mu \mathrm{M}$ and epimastigotes forms incubated with different concentrations of LQB 123 presented an inhibition of parasite growth with an $\mathrm{IC}_{50}$ of $255.1 \pm 3.6 \mu \mathrm{M}$. Finally, we investigated the mutagenic potential of the nitrone by the Salmonella/microsome assay and observed no induction of mutagenicity even in concentrations as high as $33000 \mu \mathrm{M}$. Taken together, these results present a nonmutagenic compound, with trypanocidal activity against all relevant forms of T. cruzi, offering new insights into $\mathrm{CD}$ treatment suggesting additional in vivo tests.
\end{abstract}

\section{Introduction}

Chagas disease, recognized by WHO as one of the world most important neglected tropical diseases, is a relevant socioeconomic problem in many countries [1]; it infects approximately 7 million people worldwide and is the most relevant parasitic killer in the Americas, where it is endemic [2]. T. cruzi is an obligate intracellular parasite with a complex life cycle that alternates between vertebrate host and invertebrate haematophagous triatomine insect and involves proliferative (amastigotes, epimastigotes) and nonproliferative (metacyclic, culture, or bloodstream trypomastigotes) 
stages [3]. Today, the epidemiological profile has changed due to migratory movements, which have led to both the urbanization and globalization of the disease $[4,5]$ since this illness is now observed in cities in the North of Americas and Europe where immigrants of endemic countries live nowadays $[6,7]$. From the economic perspective, the premature mortality and significant disability caused by $\mathrm{CD}$ result in a large financial impact, extending over the public healthcare system, intensifying the need for organs transplant and preventive measures such as blood screening, and monitoring the infected patients with this disease $[5,8,9]$. Today, less than $1 \%$ of people infected with $T$. cruzi have access to diagnosis and treatment [10].

Forty years after the first drugs were introduced, the chemotherapy against the etiological agent of Chagas disease remains unsatisfactory. Benznidazole (BZ) and Nifurtimox failed to control the illness in two aspects: limited efficacy for treating the chronic phase of the infection, need to be used in long-term therapy, and its high systemic toxicity including skin rashes, nausea, and kidney and liver failure. Recently, a five-year clinical trial using benznidazole (BZ) against the cardiac form of Chagas disease (BENEFIT clinical trial) demonstrated that most of $\mathrm{BZ}$ treated patients presented undetectable parasite loads, however, with no improvement on the cardiac disease [11, 12]. Additionally, Nifurtimox can also cause seizures and other nervous-system disorders [4]. Thus, the search for a more effective therapeutic and less toxic conduct becomes imperative. The general chemical structure of nitrone is $\mathrm{X}-\mathrm{CH}=\mathrm{NO}-\mathrm{Y}$, where " $\mathrm{X}$ " is a phenyl group and "Y" is a tert-butyl group (PBN). This structure has been extensively studied in many experimental and biochemical systems over the past 30 years. These nitrones were initially used in biological systems, primarily due to their "spintrap" free radicals ability [13]. Later, it was demonstrated that nitrones have low toxicity and are also suited for multiple dose regimens. Accordingly, PBN has been shown to be a relatively safe drug, even at high concentration. Despite that, the assessment of effectiveness against risk of adverse outcomes is imperative to support a clinical use [14].

There are good evidences that PBN is effective against a variety of microorganisms such as trypanosomatids. The leishmanicidal activity was also observed for the PBN derivative, C-(6-methyl-4-oxo-4H-1-benzopyran-3-yl)$\mathrm{N}$-(p-tolyl), known as NP1, which protected infected rats against the parasite by the modulation of the host immune system [15]. In 2010, Chagasic rats with cardiomyopathy, treated with an association of benznidazole and PBN, diminished oxidative damage, parasite persistence, and inflammatory pathology harmful damage. However, the same study showed that mice treated only with $\mathrm{PBN}$ presented no decline in parasite persistence, pointing out the need of chemical modifications in the PBN molecule in order to increase its trypanocidal effect [16].

Additionally, we demonstrated for the first time the protective activity of less expensive $N$-methyl nitrones against microvascular damage induced by occlusioninduced ischemia-reperfusion in the hamster cheek pouch preparation. LQB-123 was as active as or more active than $\alpha$-tocopherol, shark cartilage, and fish oil, used as references $[17,18]$ Thereafter, $O$-isoprenylated- $N$-methylarylnitrones analogues have been synthetized and the antineoplastic effects on human cancer cell lines (Jurkat and leukemia) were demonstrated [19].

Here, we evaluated the activity of a novel PBN derivate, the LQB 123, against various biological forms of Trypanosoma cruzi. Our results demonstrate the trypanocidal effect of the LQB-123 against both proliferative and infective forms of T. cruzi with no inhibition of mammalian cell viability, suggesting this compound as a promising candidate for in vivo studies.

\section{Materials and Methods}

2.1. Drug Dilution. LQB 123 was synthesized as described in Kim et al. [17] and Dias et al. [18]. Stock solutions of LQB123 were prepared in dimethyl sulfoxide (DMSO, MERCKUSA), with the final concentration of the solvent used in the experiments never exceeding $1 \%$.

\subsection{LQB 123 Activity upon Epimastigotes. Trypanosoma cruzi} $\mathrm{Y}$ strain was provided by the Trypanosomatid Collection of the Oswaldo Cruz Institute, Fiocruz, Brazil. Epimastigotes (1 $\times 10^{6}$ parasites $\left./ \mathrm{mL}\right)$ in the exponential phase of growth $(96 \mathrm{~h})$ were maintained and incubated in the presence of brain-heart infusion medium (BHI, BD Bacto, USA) supplemented with $30 \mu \mathrm{M}$ heme (Frontier Scientific, Utah, USA) and 10\% fetal calf serum (FCS, Vitrocell, Campinas, Brazil) at $28^{\circ} \mathrm{C}$. Then, increasing concentrations of LQB $123(75-1200 \mu \mathrm{M})$ were added or not to the epimastigotes in 96-well plates for $48 \mathrm{~h}$. The inhibitory concentration responsible for $50 \%$ reduction in cell viability $\left(\mathrm{IC}_{50}\right)$ was obtained after $48 \mathrm{~h}$ of treatment and determined by regression analysis of the data.

2.3. LQB 123 Activity upon Metacyclic Trypomastigotes. For in vitro differentiation, we performed the protocol determined by Contreras et al. [20] with minor modifications. Briefly, epimastigotes $\mathrm{Dm} 28 \mathrm{c}$ were grown at $28^{\circ} \mathrm{C}$ for 7 days in BHI supplemented with $30 \mu \mathrm{M}$ heme and 10\% FCS. Parasite growth was monitored by cell counting in a Neubauer chamber. Epimastigotes were harvested by centrifugation and then incubated in triatomine artificial urine (TAU) medium (190 mM NaCl, $17 \mathrm{mM} \mathrm{KCl}, 2 \mathrm{mM} \mathrm{MgCl}_{2}, 2 \mathrm{mM} \mathrm{CaCl}_{2}, 8 \mathrm{mM}$ phosphate buffer $\mathrm{pH} 6.0$ ) at a density of $5 \times 10^{8}$ cells $/ \mathrm{mL}$ for $2 \mathrm{~h}$ at $28^{\circ} \mathrm{C}$. Next, epimastigotes were diluted 1:100 (5 $\times 10^{6}$ cells $/ \mathrm{mL}$ ) in TAU3AAG medium (TAU supplemented with $10 \mathrm{mM}$ L-proline, $50 \mathrm{mM}$ L-sodium glutamate, $2 \mathrm{mM} \mathrm{L}$ sodium aspartate, and $10 \mathrm{mM} \mathrm{D-glucose).} \mathrm{After} 96 \mathrm{~h}$, culture supernatants containing metacyclic trypomastigotes were incubated in the absence or in the presence of $150 \mu \mathrm{M}$, $300 \mu \mathrm{M}, 600 \mu \mathrm{M}$, and $1200 \mu \mathrm{M}$ LQB 123 for $24 \mathrm{~h}$. The parasites viability was measured by cell counting in a Neubauer chamber. The inhibitory concentration responsible for $50 \%$ reduction in cell viability $\left(\mathrm{IC}_{50}\right)$ was obtained after $24 \mathrm{~h}$ of treatment and determined by regression analysis of the data.

2.4. Ethical Statement. All animal care and experimental protocols were conducted following the guidelines of the 
institutional care and use committee (ethics for care and use of experimental animals of IBRAG/UERJ, CEUA-UERJ) under register number CEUA/053/2012.

2.5. Effects of LQB 123 on Bloodstream Trypomastigotes Viability. Bloodstream trypomastigote forms (Y strain) were obtained from infected albino Swiss mice at the parasitaemia peak by differential centrifugation and suspended in Dulbecco modified Eagle's medium (DMEM-Life Technology) supplemented with $10 \%$ FCS. $1 \times 10^{6}$ cells/well were incubated at $37^{\circ} \mathrm{C}$ for $24 \mathrm{~h}$ in the presence of increasing concentrations of LQB $123(150-1200 \mu \mathrm{M})$. Viable trypomastigotes were counted in a Neubauer chamber. The inhibitory concentration responsible for $50 \%$ reduction in cell viability $\left(\mathrm{IC}_{50}\right)$ was obtained after $24 \mathrm{~h}$ of treatment and determined by regression analysis of the data.

2.6. Effects of LQB 123 on Intracellular Amastigotes. For infection assays, murine peritoneal macrophages were isolated from the peritoneum of Swiss Webster mice with iced DMEM medium supplemented with $10 \%$ FCS. The concentration was adjusted to $1 \times 10^{6}$ macrophages/well and incubated in at $37^{\circ} \mathrm{C}$ and $5 \% \mathrm{CO}_{2}$ for $24 \mathrm{~h}$. Nonadherent cells were removed; the cultures were washed with phosphate-buffered saline (PBS, $100 \mathrm{mM}$ phosphate buffer and $150 \mathrm{mM} \mathrm{NaCl}, \mathrm{pH}$ 7.4) and then infected with bloodstream trypomastigotes, $\mathrm{Y}$ strain (MOI 10:1). After $3 \mathrm{~h}$ of interaction, the noninternalized parasites were removed by washing with PBS. The cells were then incubated with or without LQB $123(125 \mu \mathrm{M}-$ $500 \mu \mathrm{M}$ ) in fresh DMEM for $48 \mathrm{~h}$. Cells were stained by quick Romanowsky-type stain (Panótico Rápido LB) and examined under light microscopy. The percentage of infection and the number of intracellular amastigotes were quantified using a light microscopy. The infection index was determined by the percentage of infected cells multiplied by the number of amastigotes per cell.

2.7. Toxicity to Mammalian Cells. Uninfected peritoneal macrophages $\left(2.5 \times 10^{5}\right.$ cells/well $)$ were treated with the LQB 123 for $48 \mathrm{~h}$ and their toxicity was evaluated by the alamarBlue ${ }^{\circledR}$ assay [21]. The reaction was analyzed at $570 \mathrm{~nm}$ detection, using the $600 \mathrm{~nm}$ absorbance as normalization value. The cell viability was not affected by the final DMSO concentration (data not shown).

2.8. Salmonella/Microsome Assay. Stationary growth cultures of histidine auxotrophic Salmonella typhimurium (2 $\times 10^{9}$ cells $/ \mathrm{mL}$ ) TA97, TA98, TA100, TA102, and TA1535 strains were obtained at $37^{\circ} \mathrm{C}$ in lysogenic broth $(\mathrm{LB}, 10 \mathrm{~g} / \mathrm{L}$ tryptone; $5 \mathrm{~g} / \mathrm{L}$ yeast extract; $10 \mathrm{~g} / \mathrm{L} \mathrm{NaCl}$ ) containing $8 \mu \mathrm{g} / \mathrm{mL}$ ampicillin and $2 \mu \mathrm{g} / \mathrm{mL}$ tetracycline (only for TA102). All strains used in the present work were from our laboratory collection. Survival experiments were carried out without (-S9) and with (+S9) exogenous metabolization (Moltox ${ }^{\mathrm{TM}}$, Molecular Toxicology Inc., Boone, NC) in order to determine the nontoxic concentrations to be used in the subsequent studies (data not shown). Then, the Ames test was performed according to Maron and Ames [22]. DMSO was used as a
TABLE 1: LQB 123 effect upon Trypanosoma cruzi forms.

\begin{tabular}{lccc}
\hline Parasite forms & $\mathrm{IC}_{50}(\mu \mathrm{M})$ & Time $(\mathrm{h})$ & $\mathrm{SI}$ \\
\hline $\begin{array}{l}\text { Epimastigotes (Y strain) } \\
\text { Metacyclic trypomastigotes }\end{array}$ & $255.15 \pm 32.6$ & 24 & $>15$ \\
$\begin{array}{l}\text { (Dm28c) } \\
\text { Bloodstream }\end{array}$ & $191.8 \pm 10.5$ & 24 & $>21$ \\
$\begin{array}{l}\text { trypomastigotes (Y strain) } \\
\begin{array}{l}\text { Intracellular amastigotes (Y } \\
\text { strain) }\end{array}\end{array}$ & $259.4 \pm 6.1$ & 24 & $>16$ \\
\hline
\end{tabular}

The trypanocidal activity was expressed as the $\mathrm{IC}_{50}$, corresponding to the concentration that leads to $50 \%$ parasite lysis.

$\mathrm{CC}_{50}>4000 \mu \mathrm{M}$ (drug concentration which reduced $50 \%$ of peritoneal macrophage viability).

$\mathrm{SI}$ (selectivity index) $=\mathrm{CC}_{50} / \mathrm{IC}_{50}$.

negative control. The positive controls used for assays in the absence of S9 mix were 4-nitroquinoline-1-oxide (4-NQO), at $526 \mu \mathrm{M}$ and $263 \mu \mathrm{M}$ for TA97 and TA98, respectively; sodium azide (SA) at $769 \mu \mathrm{M}$ and $154 \mu \mathrm{M}$ for TA100 and TA1535, respectively; and mitomycin $\mathrm{C}$ (MMC), at $149 \mu \mathrm{M}$; in the presence of $\mathrm{S} 9 \mathrm{mix}$ the positive controls were benzo[a]pyrene, at $396 \mu \mathrm{M}$ for TA102 and 2-aminoanthracene (2-AA), at $517 \mu \mathrm{M}$ for TA97, TA98, TA100, and TA1535. All chemicals were from Sigma Co.

2.9. Statistical Analysis. Statistical analysis was conducted with GraphPad Prism 5 software (GraphPad Software, Inc., San Diego, CA). Data were analyzed by one-way ANOVA, and differences between groups were assessed with Tukey's posttest. The level of significance was set at $p<0.05$.

\section{Results}

3.1. LQB 123 Activity against T. cruzi Bloodstream and Metacyclic Trypomastigotes and Epimastigotes. The treatment with LQB-123 was effective against epimastigotes, bloodstream, and metacyclic trypomastigotes with IC50 in the range of $75-1200 \mu \mathrm{M}$. For bloodstream trypomastigotes $\mathrm{IC}_{50} / 24 \mathrm{~h}$ it was $259.4 \pm 6.1 \mu \mathrm{M}$ and for metacyclic trypomastigotes the $\mathrm{IC}_{50} / 24 \mathrm{~h}$ was $191.8 \pm 10.5 \mu \mathrm{M}$. The mammalian proliferative form of $T$. cruzi was more susceptible to LQB 123, with $\mathrm{IC}_{50} / 48 \mathrm{~h}$ values of $188.2 \pm 47.5 \mu \mathrm{M}$ (Table 1 ). It is important to point out that $T$. cruzi is genetically classified into six intraspecies lineages, currently called discrete typing units (DTUs): TcI-VI [23]. This intraspecific diversity has been demonstrated by differences in morphology of blood forms, virulence, pathogenicity, immunological properties, infectivity in host cells, and susceptibility to chemotherapeutic agents [24]. The Dm28c clone belongs to DTU I, while Y strain belongs to DTU II. Despite that, the inhibitory concentrations for both strains or forms were similar.

3.2. LQB 123 Activity against T. cruzi Intracellular Amastigotes. Once more, LQB 123 exerted a strong inhibitory effect against another proliferative form of T. cruzi, the intracellular amastigotes. After $48 \mathrm{~h}$, the compound decreased the number of infected cells in $\mathrm{IC}_{50}$ by $78.3 \%$, the amastigotes inside each 
cell by $73.50 \%$, and $50 \%$ of the infectivity index of amastigotes $(188.2 \mu \mathrm{M})$ compared to DMSO control (Figures 1(a), 1(b), and $1(\mathrm{c}))$.

3.3. Mammalian Toxicity Assay. The cytotoxicity assay was performed to evaluate the safety of the compound in noninfected peritoneal macrophages. The cells were exposed to different concentrations of LQB 123 for $48 \mathrm{~h}$, and the concentration that was cytotoxic to $50 \%$ of the cells $\left(\mathrm{CC}_{50}\right)$ was higher than $4000 \mu \mathrm{M}$ (data not shown). Interestingly, comparing the $\mathrm{CC}_{50}$ with the $\mathrm{IC}_{50} / 48 \mathrm{~h}$, the nitrone exhibited higher Selective Index values comparing to metacyclic trypomastigotes $(>21)$ or bloodstream trypomastigotes $(>16)$, the relevant clinical forms of T. cruzi (Table 1), indicating that LQB 123 was more active against the parasite, showing no toxicity to uninfected macrophages in vitro.

3.4. Salmonella/Microsome Assay. The incubation with LQB 123 in the absence or in the presence of exogenous metabolization showed no mutagenic activity over the sensitive strains (mutagenic index $\leq 2$ ). Also, a major reduction of the induced revertants was obtained for TA97, TA98, and strains even in the absence of metabolic activation (Table 2). Moreover, the highest concentration used $(33000 \mu \mathrm{M})$, corresponding to 127 times the $\mathrm{IC}_{50}$ for bloodstream trypomastigotes, led to a significant decrease in the survival of TA100 and TA1535 strains. However, these toxic effects were not observed in the presence of exogenous metabolization (except TA100 strains in the highest concentration) indicating that S9 mix can have a detoxifying effect on the compounds responsible for bacterial growth inhibition.

\section{Discussion}

After 107 years of its discovery, the chemotherapy of this disease is still controversial and unsatisfactory, provided that the only two available drugs are toxic, possibly carcinogenic, besides presenting low effectiveness against the chronic phase of the disease [25].

Despite that, efforts have been made in order to improve CD treatment using the available drug, benznidazole; however, so far, benznidazole did not result in a statistically significant improvement in cardiac clinical outcomes [12]. The fact that $\mathrm{CD}$ is a neglected tropical disease becomes very alarming if compared with the death rates and outcomes of nonneglected illnesses such as cancer. An extrapolation calculated using the deaths of BENEFIT clinical trial patients and those collected by WHO estimates that 200.000 people will die from Chagasic cardiomyopathy over the next five years [11]. Therefore, a next generation of new products is mandatory for the establishment of novel therapies for CD. In this scenario, organic molecules generically known as nitrones have been used for more than 30 years in the analytic chemistry and in biochemistry in order to detect and establish free radicals. The PBN (alpha-phenyl-tertbutyl nitrone) is among the most studied and used for this objective, being considered an antioxidant that scavenges a wide variety of free radical species and inhibits free radical generation and also demonstrating strong pharmacological activities in disease models related to the overproduction of mitochondrial ROS, as well as neurodegenerative illnesses, stroke, and Alzheimer [13].

Wen et al. [16] demonstrated an oral administration of PBN resulting in the preservation of cardiac functions, the increase of mitochondrial function, and oxidative stress decrease in chronically infected rats by T. cruzi. However, these effects were not able to decrease the inflammatory infiltrate, or the parasite persistency.

LQB 123 compound showed effectiveness against all biologic forms of $T$. cruzi. It is also possible to highlight the trypanocidal action against infective forms, metacyclic trypomastigotes, and bloodstream, with $\mathrm{IC}_{50} / 24 \mathrm{~h} 191.8 \mu \mathrm{M}$ and $259.4 \mu \mathrm{M}$, respectively, and a higher susceptibility over the mammalian stage proliferative form $\left(\mathrm{IC}_{50} / 48 \mathrm{~h} 188.2 \mu \mathrm{M}\right)$ (Table 1). We also observed a LQB123 action in peritoneal macrophages cultures infected by this parasite. When $10: 1$ parasite/macrophage ratio was used, the LQB123 greatly diminished the percentage of infected macrophages, the intracellular amastigote number, and the decrease of infectivity index (Figure 1). Although presenting apparently elevated $\mathrm{IC}_{50}$, these nitrones had already been presented as highly tolerated molecules. In 2004, a clinical trial demonstrated the effectiveness of NXY-059 compound (another PBN derivative) in patients with ischemic stroke, even when concentrations higher than $260 \mu \mathrm{M}$ of the compound were administrated intravenously [26].

Our results also suggest that the modifications in the PBN that originated the derivate LQB 123 maintained a very low toxicity of the compound for mammalian cells. The alamarBlue viability assays showed no harmful effect in peritoneal macrophages cells, even in the presence of $4000 \mu \mathrm{M}$ of the nitrone, a concentration 21 times higher than the necessary to abolish the intracellular amastigote infection. Also, the LQB 123 compound presented greater selective action for the parasite highly infective trypomastigote forms with selectivity index higher than 16 for bloodstream trypomastigotes and higher than 21 for metacyclic trypomastigotes (Table 1). The marked selective effect of the LQB123 over intracellular amastigotes is also demonstrated in Figure $1(\mathrm{~d})$, with $500 \mu \mathrm{M}$ of the compound totally abolishing the macrophage infection, showing once more no deleterious effect to the mammalian cell.

Following the method recommended by the Organization for Economic Cooperation and Development (OECD) TG471 [27], we applied the Salmonella/microsome assay. This assay is commonly employed as an initial screening to investigate the mutagenic potential of chemicals because there is a high predictive value for rodent carcinogenicity when a mutagenic response is obtained [28, 29]. LBQ 123 induced neither frameshift nor base pair substitutions at concentrations up to $2200 \mu \mathrm{M}$, a dose that corresponds to 10 times the $\mathrm{IC}_{50}$ calculated for bloodstream trypomastigotes. The toxic effects were observed only at high concentrations (127 times the $\mathrm{IC}_{50} \mathrm{cal}$ culated for bloodstream trypomastigotes) and in the absence of exogenous metabolization, strongly indicating that S9 mix probably has a detoxifying effect of the nitrone structure, responsible for the inhibition of bacterial growth (Table 2). 


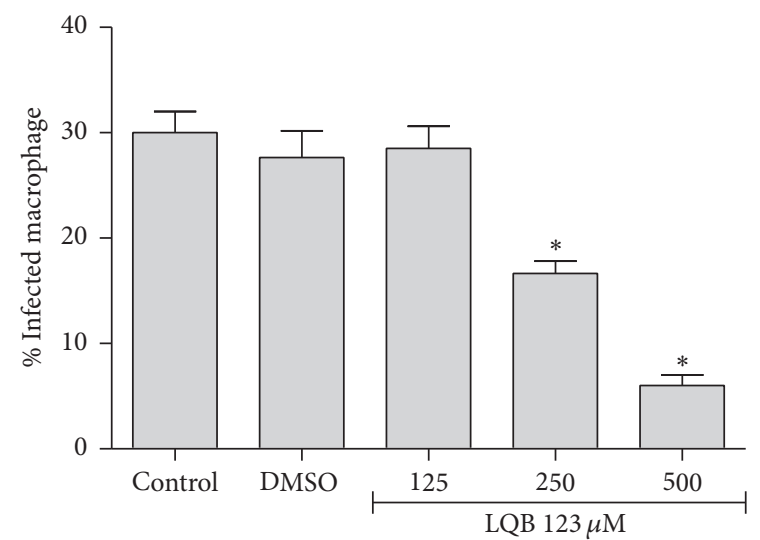

(a)

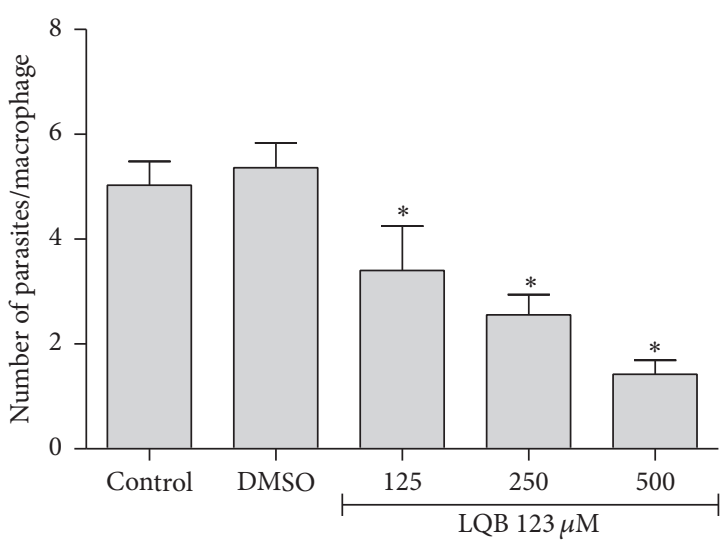

(b)

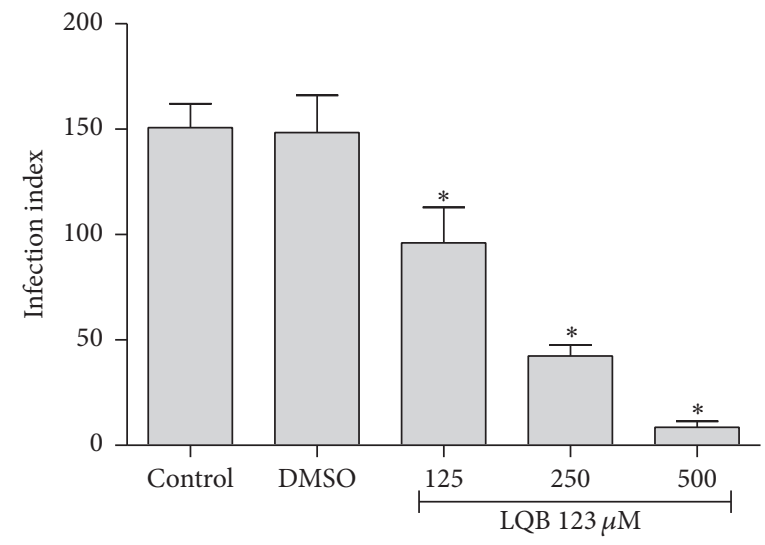

(c)

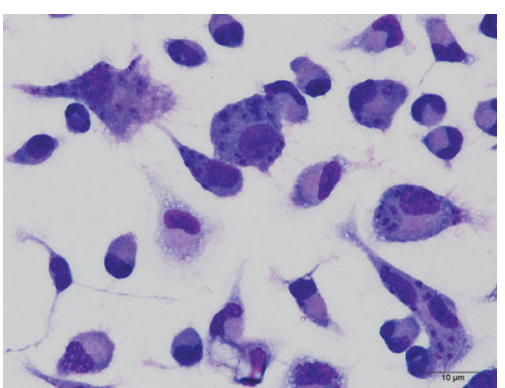

Control

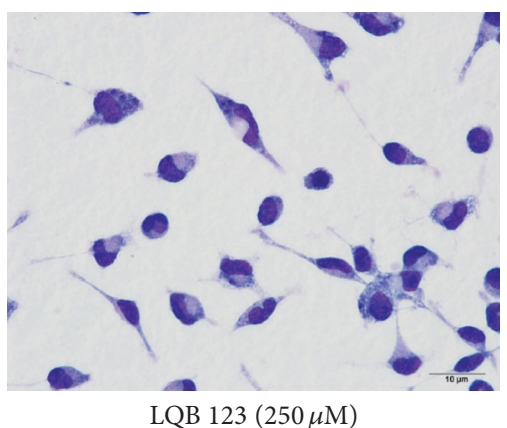

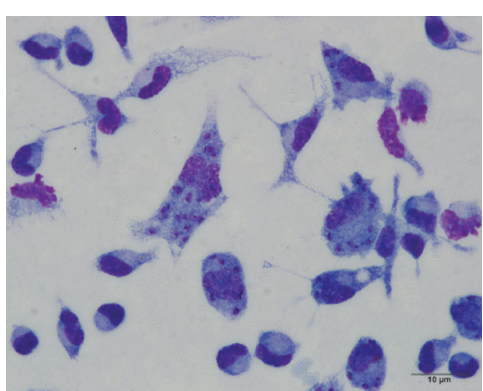

LQB $123(125 \mu \mathrm{M})$

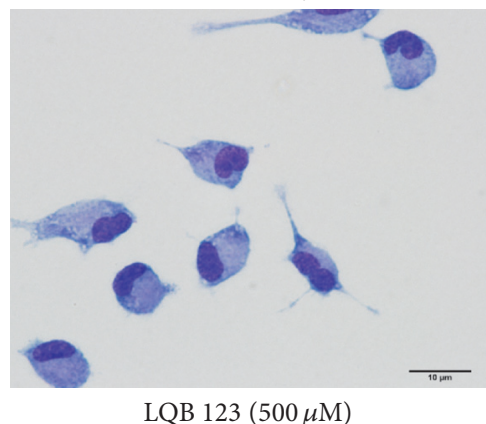

(d)

FIgURE 1: LQB 123 decreases T. cruzi infection. (a) The percentage of infection, (b) the number of parasites/macrophage, and (c) the infection index. The infection index was determined by the percentage of infected cells multiplied by the number of parasites per cell. (d) Light microscopy of T. cruzi infected macrophages after 3 days. The cells were stained by quick Romanowsky-type stain (Panótico Rápido LB) and examined under a light microscope at 100x magnification. Scale bars $=10 \mu \mathrm{m}$. The data from DMSO represents the concentration present in the highest dose of the drug. Results are shown as mean $\pm \mathrm{SD} ; *$ represents $p<0.05$ in relation to the control group by ANOVA one-way test and Tukey's posttest. Data are representative of three independent experiments performed in triplicate. 
TABLE 2: Number of Salmonella typhimurium TA97, TA198, TA100, TA1535, and TA102 revertant colonies per plate in the presence of the PBN derivate following the preincubation procedure of the Salmonella/microsome assay in the absence (-) and in the presence (+) of exogenous metabolizing system from rat liver S9-mix.

\begin{tabular}{|c|c|c|c|c|c|}
\hline \multirow{2}{*}{ Strain } & \multirow{2}{*}{ Dose $(\mu \mathrm{M})$} & \multicolumn{2}{|c|}{$-\mathrm{S} 9$} & \multicolumn{2}{|c|}{$+\mathrm{S} 9$} \\
\hline & & Mean $\pm \mathrm{SD}^{\mathrm{a}}$ & $\mathrm{MI}^{\mathrm{b}}$ & Mean $\pm \mathrm{SD}^{\mathrm{a}}$ & $\mathrm{MI}^{\mathrm{b}}$ \\
\hline \multirow{7}{*}{ TA97 } & 0 & $112 \pm 9$ & 1.0 & $166 \pm 19$ & 1.0 \\
\hline & 22 & $114 \pm 20$ & 1.0 & $192 \pm 15$ & 1.2 \\
\hline & 220 & $92 \pm 22$ & 0.8 & $192 \pm 18$ & 1.2 \\
\hline & 2200 & $97 \pm 25$ & 0.9 & $183 \pm 11$ & 1.1 \\
\hline & 22000 & $11 \pm 2^{*}$ & $0.1^{*}$ & $183 \pm 18$ & 1.1 \\
\hline & 33000 & $1 \pm 0^{*}$ & $0.0^{*}$ & $155 \pm 11$ & 0.9 \\
\hline & $\mathrm{PC}$ & $1304 \pm 226^{*}$ & 12.0 & $1488 \pm 139^{*}$ & 9.0 \\
\hline \multirow{7}{*}{ TA98 } & 0 & $24 \pm 9$ & 1.0 & $19 \pm 1$ & 1.0 \\
\hline & 22 & $31 \pm 2$ & 1.3 & $22 \pm 6$ & 1.2 \\
\hline & 220 & $29 \pm 1$ & 1.2 & $18 \pm 7$ & 1.0 \\
\hline & 2200 & $25 \pm 4$ & 1.0 & $19 \pm 1$ & 1.0 \\
\hline & 22000 & $8 \pm 3^{*}$ & $0.3^{*}$ & $18 \pm 9$ & 1.0 \\
\hline & 33000 & $7 \pm 2^{*}$ & $0.3^{*}$ & $20 \pm 2$ & 1.1 \\
\hline & $\mathrm{PC}$ & $613 \pm 32^{*}$ & 26.0 & $291 \pm 36^{*}$ & 16.0 \\
\hline \multirow{7}{*}{ TA100 } & 0 & $201 \pm 35$ & 1.0 & $272 \pm 45$ & 1.0 \\
\hline & 22 & $221 \pm 28$ & 1.1 & $242 \pm 41$ & 0.9 \\
\hline & 220 & $205 \pm 5$ & 1.0 & $274 \pm 35$ & 1.0 \\
\hline & 2200 & $154 \pm 19$ & 0.8 & $266 \pm 38$ & 1.0 \\
\hline & 22000 & $134 \pm 10^{*}$ & $0.7^{*}$ & $226 \pm 33$ & 0.8 \\
\hline & 33000 & $52 \pm 0^{*}$ & $0.3^{*}$ & $215 \pm 25$ & 0.8 \\
\hline & $\mathrm{PC}$ & $637 \pm 52^{*}$ & 3.2 & $1257 \pm 107^{*}$ & 4.6 \\
\hline \multirow{7}{*}{ TA1535 } & 0 & $13 \pm 0$ & 1.0 & $17 \pm 3$ & 1.0 \\
\hline & 22 & $15 \pm 2$ & 1.2 & $17 \pm 3$ & 1.0 \\
\hline & 220 & $14 \pm 2$ & 1.1 & $15 \pm 3$ & 0.9 \\
\hline & 2200 & $11 \pm 3$ & 0.8 & $12 \pm 1$ & 0.7 \\
\hline & 22000 & $11 \pm 2$ & 0.8 & $12 \pm 1$ & 0.7 \\
\hline & 33000 & $12 \pm 2$ & 0.9 & $12 \pm 1$ & 0.7 \\
\hline & $\mathrm{PC}$ & $615 \pm 83^{*}$ & 47.3 & $168 \pm 18^{*}$ & 10.1 \\
\hline \multirow{7}{*}{ TA102 } & 0 & $433 \pm 10$ & 1.0 & $202 \pm 12$ & 1.0 \\
\hline & 22 & $449 \pm 30$ & 1.0 & $214 \pm 16$ & 1.1 \\
\hline & 220 & $427 \pm 29$ & 1.0 & $218 \pm 8$ & 1.1 \\
\hline & 2200 & $409 \pm 45$ & 0.9 & $201 \pm 11$ & 1.0 \\
\hline & 22000 & $534 \pm 67$ & 1.2 & $181 \pm 26$ & 0.9 \\
\hline & 33000 & $533 \pm 15$ & 1.2 & $152 \pm 7$ & 0.8 \\
\hline & PC & $1638 \pm 201^{*}$ & 3.8 & $1136 \pm 78^{*}$ & 5.6 \\
\hline
\end{tabular}

${ }^{\mathrm{a}}$ Number of revertant colonies per plate: mean and standard deviation (SD) values of three replicates. ${ }^{\mathrm{b}} \mathrm{MI}$ : mutagenicity index: ratio of the number of revertant colonies induced by the samples/spontaneous number by the negative control. Positive mutagenicity (MI > 2) is indicated in bold. The sample vehicle (DMSO) was tested as a negative control. The doses of the positive controls (PC) in assays without S9: $526 \mu \mathrm{M}$ and $263 \mu \mathrm{M}$ of 4-NQO (TA97 and TA98, resp.), 769 $\mu \mathrm{M}$ and $154 \mu \mathrm{M}$ of AS (TA100 and TA1535, resp.), and $149 \mu \mathrm{M}$ of MMS (TA102). In assays with S9:396 $\mu \mathrm{M}$ of B $[\alpha] \mathrm{P}$ (TA102) and 517 $\mu \mathrm{M}$ of 2-AA (for others strains), statistically significant differences $\left({ }^{*} p<0.05\right)$ relative to the negative control by ANOVA and Tukey's test are indicated.

Another possible chemotherapy applicability of LQB 123 is further enhanced because the benznidazole mutagenic activity was tested by Ames assay [30]. The maximum mutagenic rate of benznidazole was obtained at 100 micrograms per plate (20 times $\mathrm{IC}_{50}$ calculated for bloodstream trypomastigotes). This concentration when applied to LQB 123 presents no mutagenic effect.

Thus, it is plausible to suggest that the LQB 123 molecule retained some of the chemical properties of $\mathrm{PBN}$, such as the low toxicity and a prominent trypanocidal effect in vitro. 
It is important to point out that the the beneficial effect of $\mathrm{PBN}$ in vivo against $\mathrm{CD}$ was a consequence of its free radical scavenging properties; however, recent reports have shown that the antileukemic properties of the PBN derivate, the Ogeranylated nitrone (LQB-278), were not free radical related but a consequence of cell cycle regulation by the inhibition of p21 expression [19]. Therefore, the antioxidant property may not be the only mechanism of action of these molecules. Thus, further experiments using biochemical and molecular approaches are needed to better characterize the mechanism of action of the LQB123. Several well-known examples of different modes of action of closely related analogues can be found in medicinal chemistry. So, gradually PBN or LQB 123 changes from a more specific trypanocidal compound effect are necessary.

Finally, it is important to establish that a desired compound should have trypanocidal activity without inducing toxic effects to cells at concentrations that can be achieved in vitro. The relative effectiveness of an investigational product in inhibiting parasite survival compared to inducing cell death and the therapeutic or selectivity index has to be considered. Thus, the selection of new trypanocidal compounds should take into account a high selectivity index giving maximum trypanocidal activity with minimal cell toxicity.

Also, the new therapies to be established should include new pediatric formulations of the available drug, benznidazole, and new benznidazole treatment regimens (including combination therapies), as well as a new therapeutic vaccine linked to benznidazole chemotherapy [31].

\section{Conclusions}

Taken together, these results broaden new perspectives for the Chagas disease treatment once LQB 123, a novel nitrone derivate, significantly decreased mammalian cells infections by T. cruzi. Furthermore, the low toxicity of LQB 123 against the vertebrate cells, proven in this work, is a very important result that justifies future animal experimentation, as well as the testing of new PBN analogues.

\section{Competing Interests}

The authors report no conflict of interests in this work.

\section{Acknowledgments}

The authors would to thank Marcos Meuser Batista for the help with bloodstream trypomastigotes acquisition.

\section{References}

[1] P. J. Hotez, D. H. Molyneux, A. Fenwick et al., "Control of neglected tropical diseases," New England Journal of Medicine, vol. 357, no. 10, pp. 1018-1027, 2007.

[2] WHO-World Health Organization 2015 Chagas disease (American trypanosomiasis), http://www.who.int/mediacentre/factsheets/fs340/en/.

[3] W. De Souza, "Cell biology of Trypanosoma cruzi," International Review of Cytology, vol. 86, pp. 197-283, 1984.
[4] J. R. Coura and P. A. Viñas, "Chagas disease: a new worldwide challenge," Nature, vol. 465, no. 7301, pp. S6-S7, 2010.

[5] C. J. Perez, A. J. Lymbery, and R. C. A. Thompson, "Reactivation of chagas disease: implications for global health," Trends in Parasitology, vol. 31, no. 11, pp. 595-603, 2015.

[6] C. Bern, S. Kjos, M. J. Yabsley, and S. P. Montgomery, "Trypanosoma cruzi and chagas' disease in the united states," Clinical Microbiology Reviews, vol. 24, no. 4, pp. 655-681, 2011.

[7] G. A. Schmunis and Z. E. Yadon, "Chagas disease: a Latin American health problem becoming a world health problem," Acta Tropica, vol. 115, no. 1-2, pp. 14-21, 2010.

[8] C. Franco-Paredes, A. Von, A. Hidron et al., "Chagas disease: an impediment in achieving the Millennium Development Goals in Latin America," BMC International Health and Human Rights, vol. 7, article 7, 2007.

[9] M. C. Nunes, W. Dones, C. A. Morillo, J. J. Encina, and A. L. Ribeiro, "Council on chagas disease of the interamerican society of cardiology chagas disease: an overview of clinical and epidemiological aspects," Journal of the American College of Cardiology, vol. 62, no. 9, pp. 767-776, 2013.

[10] I. Ribeiro, A.-M. Sevcsik, F. Alves et al., "New, improved treatments for Chagas disease: from the R\&D pipeline to the patients," PLoS Neglected Tropical Diseases, vol. 3, no. 7, article no. e484, 2009.

[11] B. Pecoul, C. Batista, E. Stobbaerts et al., "The BENEFIT trial: where do we go from here?" PLoS Neglected Tropical Diseases, vol. 10, no. 2, Article ID e0004343, 2016.

[12] C. A. Morillo, J. A. Marin-Neto, A. Avezum et al., "Randomized trial of benznidazole for chronic chagas' cardiomyopathy," New England Journal of Medicine, vol. 373, no. 14, pp. 1295-1306, 2015.

[13] R. A. Floyd, R. D. Kopke, C.-H. Choi, S. B. Foster, S. Doblas, and R. A. Towner, "Nitrones as therapeutics," Free Radical Biology and Medicine, vol. 45, no. 10, pp. 1361-1374, 2008.

[14] R. A. Floyd, R. A. Towner, T. He, K. Hensley, and K. R. Maples, "Translational research involving oxidative stress and diseases of aging," Free Radical Biology and Medicine, vol. 51, no. 5, pp. 931-941, 2011.

[15] S. Mallick, S. Halder, A. Dutta et al., "Chromone linked nitrone derivative induces the expression of iNOS2 and Th1 cytokines but reduces the Th2 response in experimental visceral leishmaniasis," International Immunopharmacology, vol. 15, no. 4, pp. 772-779, 2013.

[16] J.-J. Wen, S. Gupta, Z. Guan et al., "Phenyl- $\alpha$-tert-butyl-nitrone and benzonidazole treatment controlled the mitochondrial oxidative stress and evolution of cardiomyopathy in chronic chagasic rats," Journal of the American College of Cardiology, vol. 55, no. 22, pp. 2499-2508, 2010.

[17] S. Kim, G. V. M. D. A. Vilela, J. Bouajila et al., " $\alpha$-PhenylN-tert-butyl nitrone (PBN) derivatives: synthesis and protective action against microvascular damages induced by ischemia/reperfusion," Bioorganic \& Medicinal Chemistry, vol. 15, no. 10, pp. 3572-3578, 2007.

[18] A. G. Dias, C. E. V. Santos, F. Z. G. A. Cyrino, E. Bouskela, and P. R. R. Costa, "N-tert-Butyl and N-methyl nitrones derived from aromatic aldehydes inhibit macromolecular permeability increase induced by ischemia/reperfusion in hamsters," Bioorganic and Medicinal Chemistry, vol. 17, no. 11, pp. 3995-3998, 2009.

[19] D. S. S. Costa, T. Martino, F. C. Magalhães et al., "Synthesis of N-methylarylnitrones derived from alkyloxybenzaldehydes and antineoplastic effect on human cancer cell lines," Bioorganic and Medicinal Chemistry, vol. 23, no. 9, pp. 2053-2061, 2015. 
[20] V. T. Contreras, T. C. Araujo-Jorge, M. C. Bonaldo, N. Thomaz, and H. S. Barbosa, "Biological aspects of the Dm 28c clone of Trypanosoma cruzi after metacyclogenesis in chemically defined media," Memórias do Instituto Oswaldo Cruz, vol. 83, no. 1, pp. 123-133, 1988.

[21] A. Roman, D. Nawrat, and I. Nalepa, "Chronic treatment with electroconvulsive shock may modulate the immune function of macrophages," Journal of ECT, vol. 24, no. 4, pp. 260-267, 2008.

[22] D. M. Maron and B. N. Ames, "Revised methods for the Salmonella mutagenicity test," Mutation Research/Environmental Mutagenesis and Related Subjects, vol. 113, no. 3-4, pp. 173215, 1983.

[23] B. Zingales, M. A. Miles, D. A. Campbell et al., "The revised Trypanosoma cruzi subspecific nomenclature: rationale, epidemiological relevance and research applications," Infection, Genetics and Evolution, vol. 12, no. 2, pp. 240-253, 2012.

[24] S. M. F. Murta and A. J. Romanha, "Characterization of Trypanosoma cruzi," Memorias do Instituto Oswaldo Cruz, vol. 94, no. 1, pp. 177-180, 1999.

[25] S. R. Wilkinson and J. M. Kelly, “Trypanocidal drugs: mechanisms, resistance and new targets," Expert Reviews in Molecular Medicine, vol. 11, article no. e31, 2009.

[26] K. R. Maples, A. R. Green, and R. A. Floyd, "Nitrone-related therapeutics: potential of NXY-059 for the treatment of acute ischaemic stroke," CNS Drugs, vol. 18, no. 15, pp. 1071-1084, 2004.

[27] OECD, "1997 OECD Guideline for Testing of Chemicals (TG471): Bacterial Reverse Mutation Test," http://www.oecd .org/chemicalsafety/risk-assessment/1948418.pdf.

[28] K. Mortelmans and E. Zeiger, "The Ames Salmonella/microsome mutagenicity assay," Mutation Research-Fundamental and Molecular Mechanisms of Mutagenesis, vol. 455, no. 1-2, pp. 29-60, 2000.

[29] J. McCann, E. Choi, E. Yamasaki, and B. N. Ames, "Detection of carcinogens as mutagens in the Salmonella/microsome test: assay of 300 chemicals," Proceedings of the National Academy of Sciences of the United States of America, vol. 72, no. 12, pp. 51355139, 1975.

[30] R. C. Ferreira and L. C. Ferreira, "Mutagenicity of nifurtimox and benznidazole in the Salmonella/microsome assay," Brazilian Journal of Medical and Biological Research, vol. 19, no. 1, pp. 19-25, 1986.

[31] "Drugs for Neglected Diseases Initiative DNDi R\&D Projects," http://www.dndi.org/diseases-projects/portfolio/. 

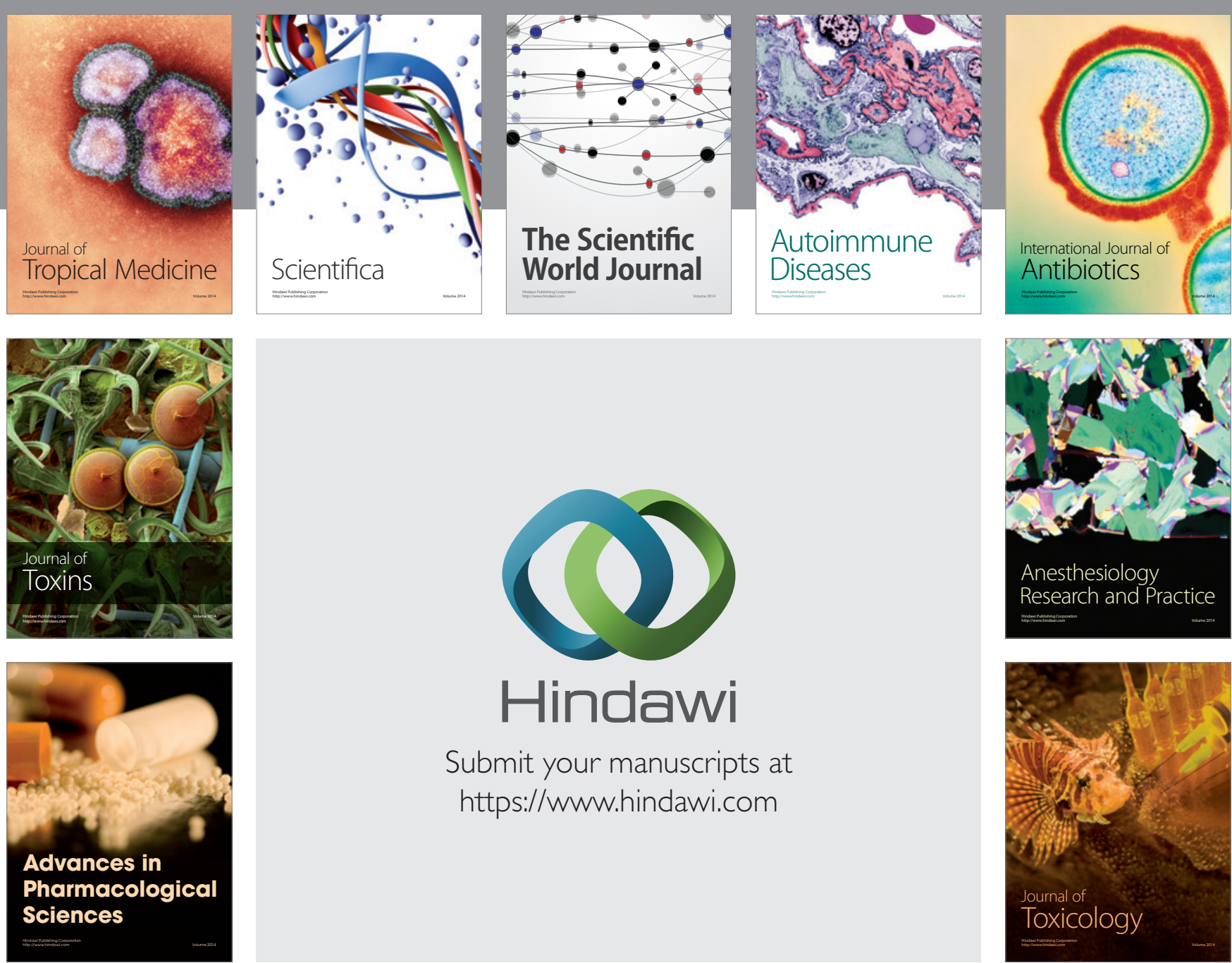

\section{Hindawi}

Submit your manuscripts at

https://www.hindawi.com
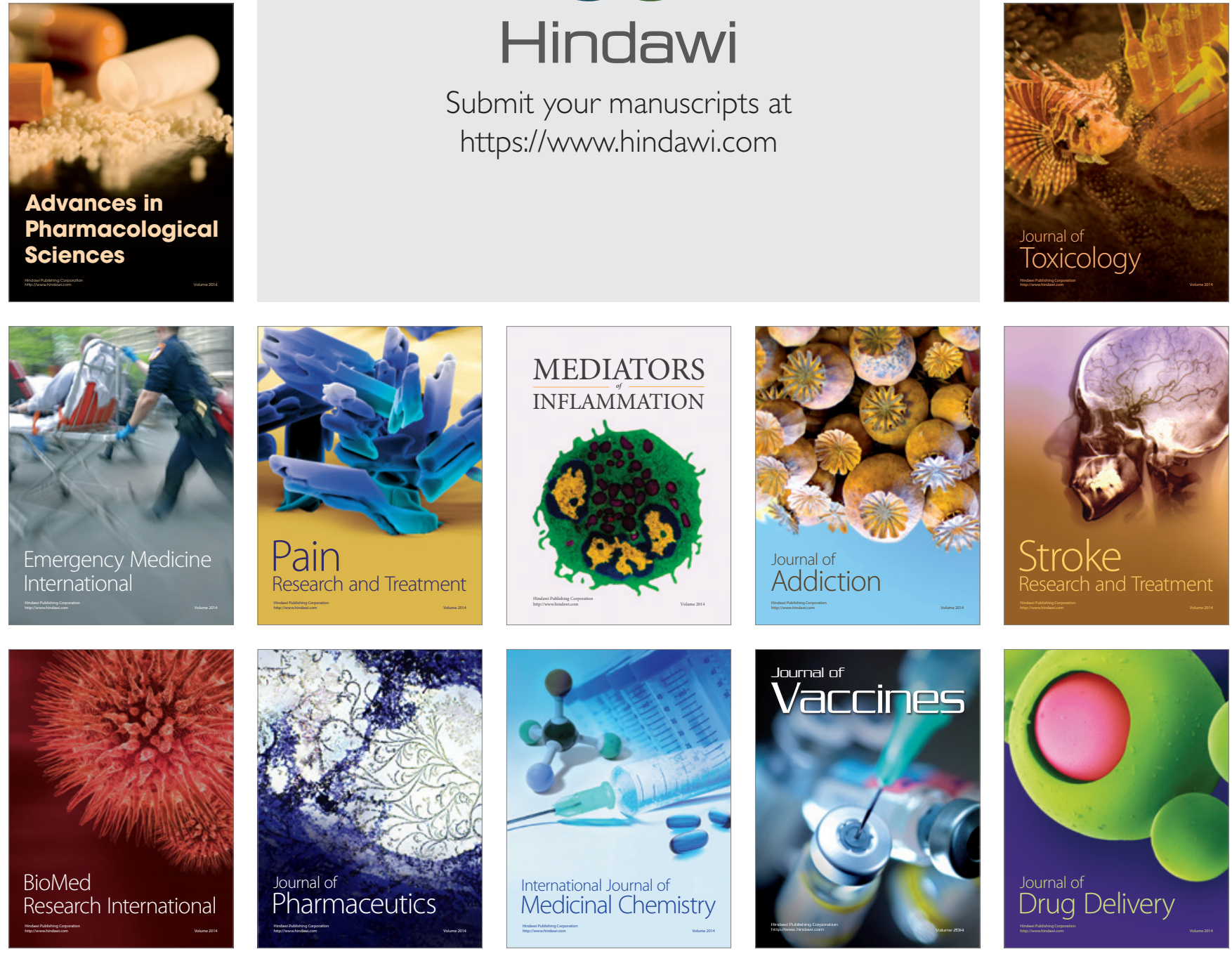